\title{
L'application du sonar à balayage latéral à la reconnaissance des fonds d'un chenal de navigation : le cas de la Seine, estuaire aménagé.
}

\author{
Patrick Lesueur, Sandric Lesourd, Jean-Paul Auffret, Nicole Poupinet \\ UPRES A-CNRS 6143 "Morphodynamique Continentale et Côtière" \\ Université de Caen-Basse Normandie
}

\begin{abstract}
Résumé
L'étude des fonds du chenal de navigation de la Seine au sonar latéral revêt une approche innovante dans la reconnaissance des sédiments superficiels de cet estuaire. En dépit des faibles profondeurs d'eau et en raison de la largeur restreinte du chenal aménagé, cette prospection a permis de réaliser une cartographie complète du chenal de navigation, de l'embouchure à Rouen. L'affleurement de fonds fossiles et la minceur de la couverture mobile sur une large portion amont du chenal ont été mis en évidence. Ailleurs, des figures sédimentaires de charriage apparaissent au fond du chenal. L'activité anthropique apparaît : empreintes des dragages, aménagement des berges, ancrages de bouées, épaves, traces de pêche au chalut dans la partie aval, sont nettement perceptibles et peuvent avoir localement une influence dans la distribution des faciès sédimentaires.
\end{abstract}

\begin{abstract}
Side-Scan Sonar and sediment sampling have been employed in order to investigate and to map the distribution of sediments and bedforms within the Seine estuary, over a distance of $120 \mathrm{~km}$ up to the outlet. A major result of this study is the thinness of the surficial sediments in a large upstream part of the estuary and the complexity in their relationships. Moreover, sonographs show bedforms in sands and gravels, instabilities, as slides in the channel slopes and give prominence to human impact, including dredging tracks and wrecks.

\section{Introduction}

A la fin des années 1970 , les processus hydrosédimentaires dans la partie aval de l'estuaire de la Seine ont fait l'objet d'études approfondies, synthétisées par Avoine (1981). Les sédiments de la Baie de Seine ont été cartographiés plus tard par la méthode du sonar à balayage latéral (Auffret et d'Ozouville, 1986). Néanmoins, jusqu'au Programme Seine-Aval (1996-99), les caractéristiques de la couverture sédimentaire de l'estuaire à l'amont de Tancarville n'avaient jamais fait l'objet d'une reconnaissance approfondie. Dans la problématique de ce programme, basée sur la connaissance du transfert des contaminants - pour partie associés aux particules fines - les zones de dépôt temporaire ou permanent des vases devaient être identifiées. La répartition des sédiments superficiels du domaine côtier étant par nature très variable, en particulier dans les estuaires, les
\end{abstract}


seuls échantillonnages ponctuels ne pouvaient prétendre répondre au problème posé. Nous avons donc entrepris une reconnaissance au sonar à balayage latéral, qui offre la possibilité d'obtenir une cartographie en continu des fonds. Cette méthode est désormais couramment utilisée : d'abord en domaine marin depuis près de quarante ans (Belderson et al., 1972), plus récemment en milieu estuarien (d'Ollier, 1979 ; Harris \& Collins, 1984) ou lacustre (Duck et McManus, 1990). Performante en milieu ouvert (Berné et al., 1986), son exploitation a été plus rarement décrite dans un fleuve aménagé, canalisé et soumis à marée comme c'est le cas ici. Nous présentons l'intérêt de cette méthode géophysique pour l'utilisation et l'entretien d'un chenal de navigation : dynamique sédimentaire et identification de "points durs".

\section{Rappel succinct du principe de prospection}

Le sonar à balayage latéral est un moyen d'exploration qui est basé sur l'émission d'ondes acoustiques par deux transducteurs à partir d'un appareillage tracté (le "poisson"), sous la forme d'un faisceau oblique (Fig. 1). Ces ondes se propagent vers le fond de l'eau à partir de ces deux transducteurs, symétriquement de part et d'autre du navire tracteur, jusqu'à la rencontre d'un obstacle correspondant aux fonds et/ou à des obstacles. La réflexion des ondes s'opère selon différentes directions, mais seule l'onde réfléchie suivant le même axe que l'onde incidente est reçue par chaque transducteur, qui joue alors le rôle de récepteur. Le signal rétrodiffusé est enregistré et visualisé sous forme de lignes successives juxtaposées suivant le déplacement du navire. Il en résulte une image (sonogramme), comparable à une photographie en vue oblique des fonds, qui aurait été réalisée avec un éclairage rasant. Les échofaciès (traces sur l'enregistrement) sont d'autant plus sombres que le milieu est rétrodiffusant : les sédiments grossiers et denses (graviers et galets) apparaissent plus sombres que les

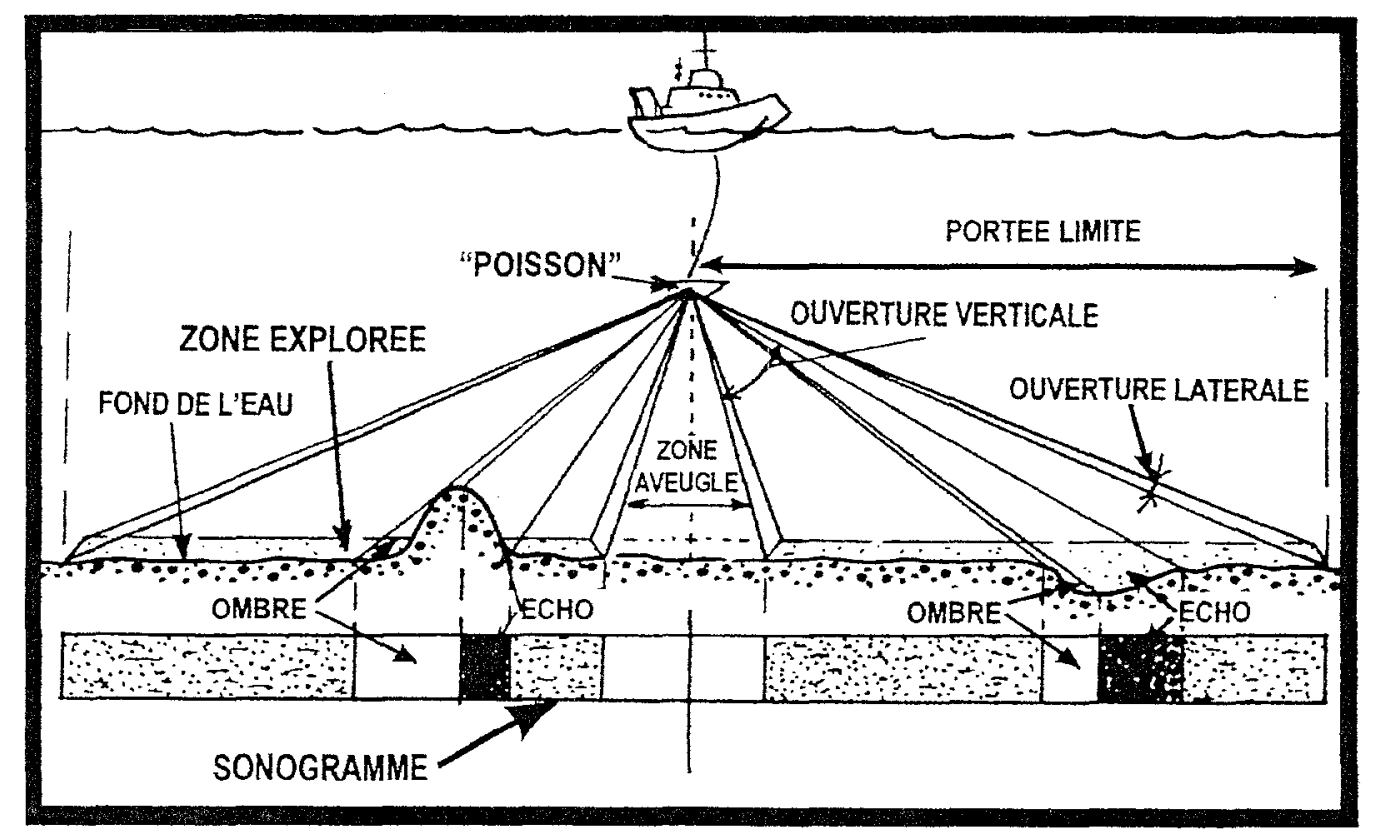

Figure 1. Principe du sonar latéral 
sables moyens et grossiers, qui eux-mêmes sont généralement plus gris que les sablons et les vases, apparaissant en blanc sur les sonogrammes.

Le sonar à balayage latéral donne donc des informations capitales sur : (1) la nature du sédiment ou la présence de rochers, par l'intensité de l'écho réfléchi, (2) sur sa morphologie, par la présence de zones où aucun écho n'apparaît et qui correspondent à des zones d'ombre acoustique, (3) sur la présence d'objets sur le fond.

\section{Moyens et déroulement de l'étude}

\subsection{Moyens nautiques}

La prospection a été réalisée du 2 au 6/12/96 par morte-eau (coefficients 38-51) mais en période de crues (1100-1200 $\left.\mathrm{m}^{3} \mathrm{~s}^{-1}\right)$. Les moyens de navigation ont été ceux de l'INSU/CNRS, le faible tirant d'eau du navire utilisé ("Côte de Normandie") permettant de longer les berges estuariennes. Le navire était équipé d'un système de positionnement de type GPS fonctionnant en mode différentiel, le déplacement du navire, de l'ordre de 4 à 5 noeuds, étant enregistré par un positionnement toutes les minutes. La profondeur d'eau sous le navire était accessible à tout instant grâce à un sondeur bathymétrique équipé d'un enregistreur à bandes. Outre l'intérêt de permettre une cartographie en continu des fonds, la méthode de prospection au sonar à balayage latéral présente l'avantage d'être relativement rapide : la surface couverte était dans notre cas de l'ordre de 1,5 à 2 $\mathrm{km}^{2}$ à l'heure.

Après un premier dépouillement des sonogrammes, la calibration nécessaire des images a été réalisée dans les mois suivant la prospection : plus de 400 échantillons (bennes, carottages) ont été prélevés et de la vidéo sous-marine a été utilisée.

\subsection{Matériel de prospection}

L'appareillage mis en œuvre par Genavir, était de type Dowty-Widescan II. Sa fréquence d'émission est de $100 \mathrm{kHz}$. Le poisson, équipé de deux transducteurs était remorqué derrière le bateau avec un décalage d'une vingtaine de mètres par rapport aux données de positionnement liées à la situation de l'antenne GPS. La portée latérale était de $100 \mathrm{~m}$ de part et d'autre du poisson. Toutes les corrections telles que celles intéressant l'obliquité des ondes acoustiques sont réalisées par la mesure. La hauteur du poisson au-dessus du fond et la compression-dilatation longitudinale de l'image (vitesse du poisson par rapport au fond) sont calculées en temps réel (la vitesse de déroulement du papier fonction de la vitesse du bateau). L'enregistrement numérique est effectué sur support magnétique par l'utilisation du logiciel Delph.

\subsection{Principe des enregistrements}

Un sonogramme comprend les informations suivantes (Fig. 2, du haut vers le bas) : (1) la hauteur d'eau (h) sous le "poisson", visualisée par une bande latérale grise, dont la largeur est d'autant plus importante que la hauteur d'eau est 
importante (ligne repère constante à 10m), (2) "l'image" (deux fois $100 \mathrm{~m}$ de large), séparée à mi-distance par une bande correspondant au signal d'émission ( $\mathrm{s}$; celle-ci est plus ou moins large selon la qualité du signal et correspond à la zone "aveugle" située à l'aplomb du poisson, (3) un repère-temps $(t)$ toutes les minutes et des lignes d'échelle (e) parallèles à la trace. Le sonogramme est retranscrit sous une forme orthonormée. Ainsi, les dimensions des objets analysés peuvent être aisément évaluées ; la définition, variable sur la largeur balayée, peut être peu inférieure à $1 \mathrm{~m}$.

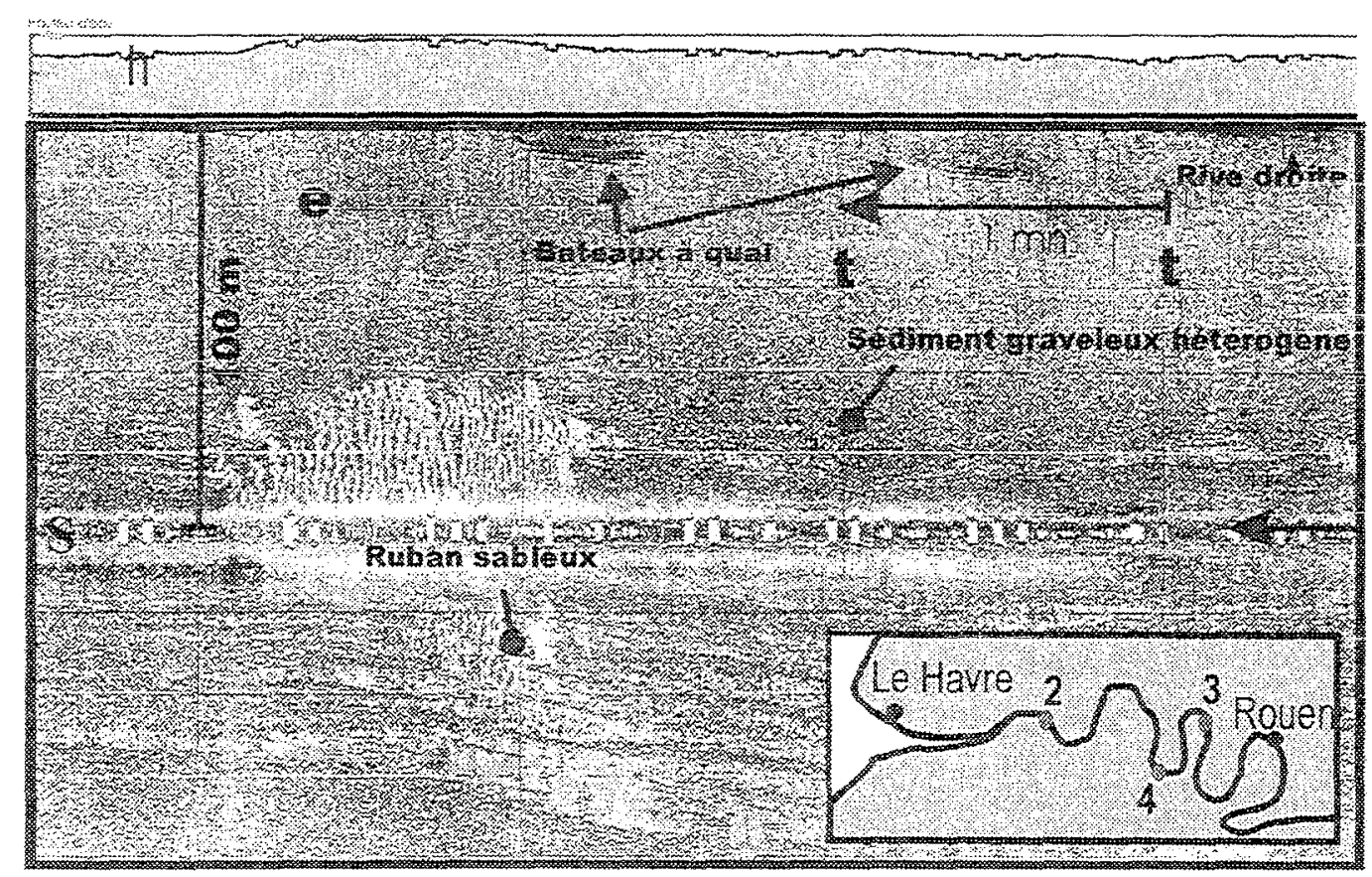

Figure 2 : Exemple-type d'informations disponibles sur les sonogrammes étudiés (Port Jérôme, pK 331,4). En encadré, position des sonogrammes (numéros de figure)

Les marques du transport par charriage sur les fonds, ainsi que l'empreinte de certaines activités anthropiques, sont ainsi clairement rendues. Un inventaire de diverses images obtenues au sonar latéral a été réalisé (Belderson et al., 1972), puis affiné pour différents potentiels d'alimentation en sable (Belderson et al., 1982).

\section{Principaux résultats}

\subsection{L'information sur les sédiments superficiels du chenal}

\subsubsection{Faciès sédimentaires}

Les faciès acoustiques sont généralement très variables sur de courtes distances. Ceci est dû (1) à la grande diversité des fonds (lithologie) et des figures sédimentaires associées à un chenal de faible largeur, qui induit une dynamique 
d'écoulement complexe dans un régime à méandres imposé, mais aussi (2) à son aménagement depuis le dernier siècle.

Les échofaciès et leur calibration par les échantillonnages ont permis de distinguer : (1) des dépôts consolidés quaternaires (argile très compacte, tourbe, tuf calcaire) et la craie ponctuellement à l'affleurement, parfois décalée par des failles, (2) des sédiments mobilisables comprenant des graves hétérogènes (graviers et galets mélangés à une large fraction sableuse), des sables homogènes mobiles formant des corps sédimentaires ou des placages sur des graviers, (3) de rares dépôts vaseux, le plus souvent en situation de dépôt transitoire (en drapage sur des sables ou sous forme de galets mous).

\subsection{2. les figures sédimentaires}

Les figures sédimentaires liées à la dynamique de la masse d'eau, indicatrices du déplacement des sédiments au fond (courants et houle à l'aval), comprennent (1) des figures de charriage et (2) des figures d'érosion du fond ou des berges immergées.

Les figures longitudinales sont fréquentes dans un écoulement canalisé comme celui de la Seine aval : (1) des rubans sableux rectilignes de haute énergie qui témoignent du déplacement des sables sur les fonds grossiers ou fossiles, (2) des sillons d'érosion du substrat sableux ou graveleux, (3) des queues de comète provoquées par des objets au fond, (4) des placages sableux, accumulations de faible épaisseur et d'orientation mal définie. Il est intéressant de noter que ces figures correspondent toujours typiquement à un milieu à fort déficit d'alimentation en sable (Belderson et al., 1982). Des figures transverses, ici des rides ou des mégarides, d'amplitude métrique et de longueur d'onde variable (quelques $m$ à quelques dizaines de $\mathrm{m}$ ) modèlent les rubans sableux.

Les sonogrammes montrent que de Rouen à Tancarville et dans les condiiions de l'étude (morte eau et crue), les figures et corps sédimentaires de charriage polarisés (tels mégarides et queues de comètes) indiquent presque toujours un sens de transport vers l'aval.

Des figures d'érosion ou d'affouillement des fonds et des pentes de berges, en particulier au niveau des rives externes des méandres sont imputées à la turbulence inhérente au courant du fleuve, probablement augmentées par la navigation. A l'aval (chenal nord de l'embouchure), des figures d'érosion sont identifiées dans des faciès vaseux relativement consolidés. Un faciès acoustique chaotique, qui correspond à l'affleurement de fonds fossiles compacts (argiles, silts, tourbes et tufs) est largement répandu entre Caudebec et Rouen. Des blocs et des cailloutis composent fréquemment ces fonds. Localement, des figures de glissement de pentes de berges immergées, sont comparables à celles identifiées en milieu aérien ou lacustre (cicatrices d'arrachement, loupes de glissement).

\subsection{Empreinte de l'action anthropique sur les fonds étudiés}

A l'extrémité amont de la zone d'étude, près de Rouen, des échos particuliers sont à rattacher aux activités portuaires. Il apparait ainsi des blocs de 
plusieurs mètres, immergés et des traces de ripage d'ancre dans le sédiment. Plus en l'aval, le profilage et la rectification de la pente des berges, pour une amélioration du chenal ou d'un accès à quai, sont parfois remarqués (ruptures de pentes). Quelques épaves sont localisées en bordure du chenal. Les points d'ancrage des bouées sont souvent nettement visibles.

Les traces d'activité anthropique les plus courantes et les plus évidentes sont dues aux dragages d'entretien et d'amélioration des profondeurs. Ainsi, en un certain nombre de lieux bien identifiés (Delouis, 1997) du chenal, des empreintes des becs d'élinde des dragues aspiratrices apparaissent nettement, comme des sillons rectilignes dans le sédiment. Au nord de l'embouchure, un faciès hétérogène tacheté de sables, correspond aux résidus de clapage de matériaux dragués dans le chenal (zone de dépôts du Kannick).

Enfin, des traces de chalutages ont été remarquées dans l'embouchure.

\subsection{Commentaire d'exemples d'enregistrements}

Trois exemples caractéristiques sont choisis.

Le premier exemple de sonogramme (Port Jérôme, pK 331.4, fig. 2) montre un chenal tapissé de sédiments hétérogènes, graveleux, portant quelques traces de dragages ou de ripages d'objets (ancres ?), partiellement estompés par la dynamique des rubans sableux caractéristiques de la haute énergie de ce secteur. Le sens du transit par charriage du sédiment, vers l'aval (à gauche sur l'enregistrement), est indiqué par l'asymétrie des mégarides en rateau et par l'orientation de queues de comètes de faible dimension.

Figure 3. Exemple de sonogramme des environs de Ambourville

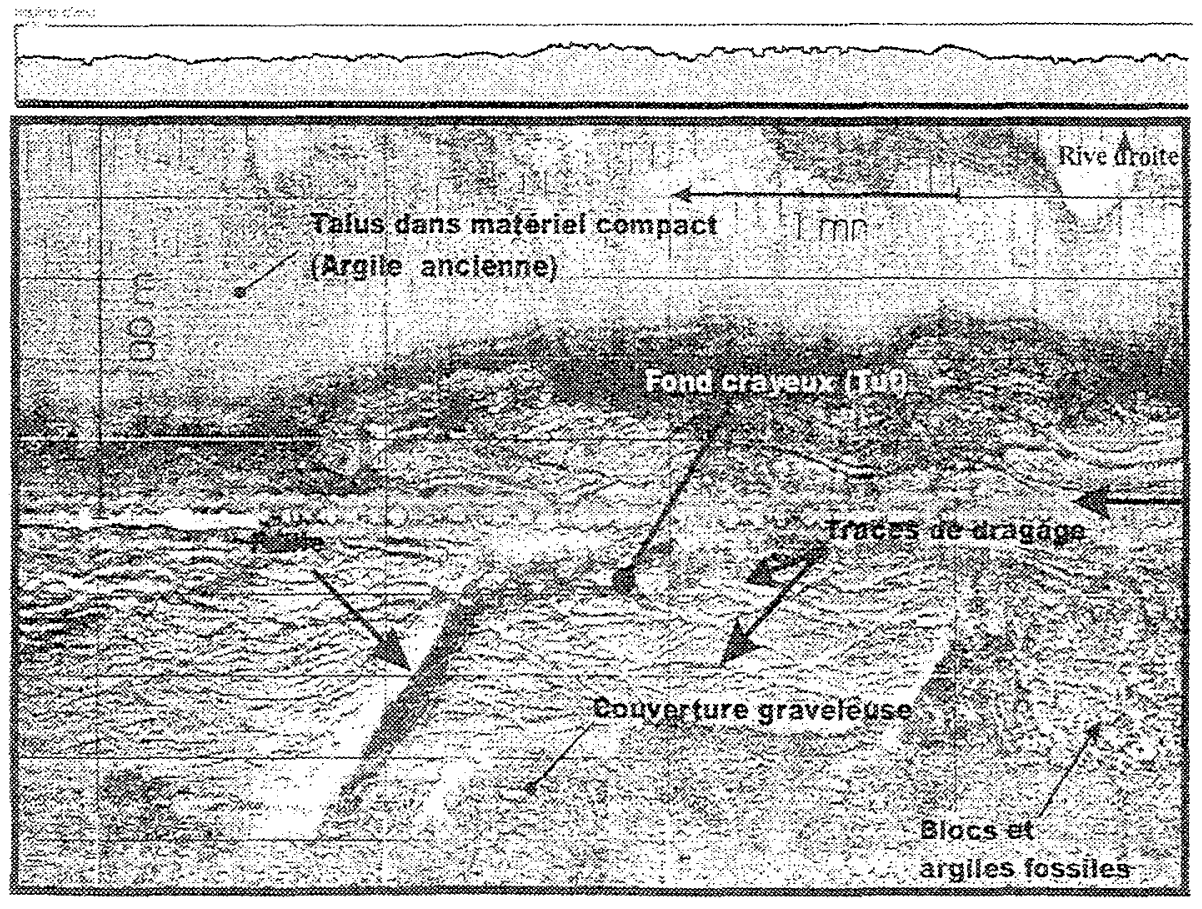


Le deuxième exemple de sonogramme présenté (Fig. 3) est typique d'une zone de seuil rocheux dans le chenal de navigation, à Ambourville (pK 273). La partie supérieure (rive droite) montre un talus dans un matériel homogène argileux. La partie centrale à inférieure montre des fonds tourmentés ; une surface claire est marquée de sillons orientés dans la direction du chenal : il s'agit de rocher crayeux, affecté de deux failles subparallèles. Ces fonds ont posé des problèmes pour la navigation : ils montrent une importante densité de traces de dragages. La couverture graveleuse apparaît à la limite inférieure de l'enregistrement, qui montre aussi (en bas, à droite) le faciès déchiqueté reconnu sur des fonds compacts fossiles affleurant sous forme de blocs.

Un autre exemple (Barneville, pK 290) montre en partie supérieure de l'enregistrement (rive gauche) un aspect uniforme de teinte gris sombre qui

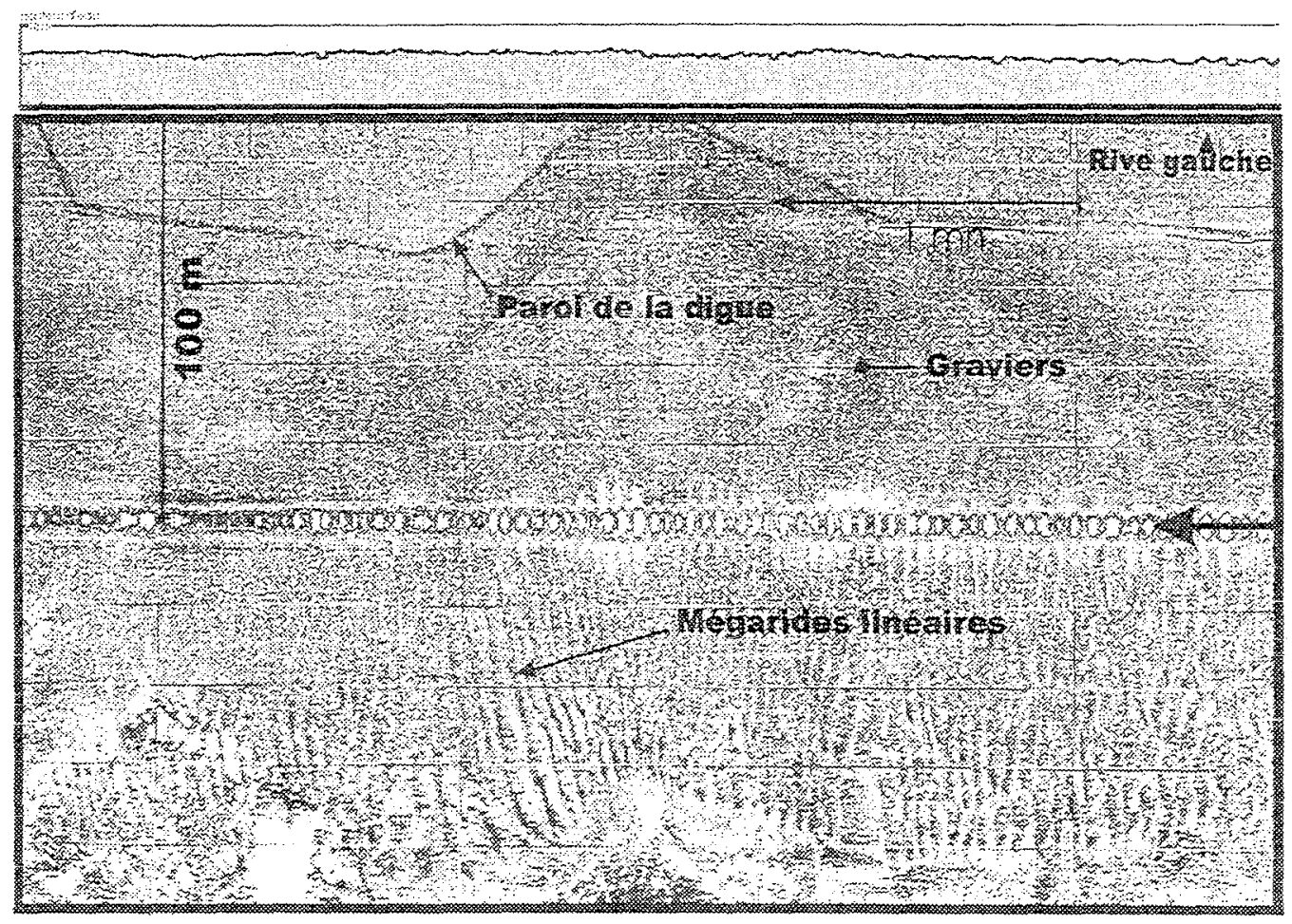

Figure 4. Exemple de sonogramme dans les environs de Barneville

correspond au faciès représentatif de ce secteur : des graviers (Fig. 4). La trace plus sombre est un écho parasite de la paroi de digue de rive gauche (berge concave du méandre). La partie inférieure du cliché montre l'amorce de barre interne de méandre (berge convexe) avec des mégarides linéaires (longueur d'onde de $4-5 \mathrm{~m}$, amplitude de l'ordre du mètre). La sinuosité d'ensemble est artificielle et provoquée par la variation de cap du navire.

\section{Conclusions}

Un des résultats essentiels de la prospection au sonar latéral des fonds de la 
Seine à l'aval de Rouen est la mise en évidence de la faible épaisseur de la couverture sédimentaire meuble et l'affleurement fréquent de dépôts fossiles au fond d'une part importante du chenal. Selon le lieu, affleurent des galets et des graves fluviatiles, des argiles et des silts anciens très compacts, des tourbes, des tufs calcaires, voire du rocher crayeux ou marneux altéré. Des seuils marqués dans les fonds indurés existent parfois au plafond du chenal de navigation. Fréquents à l'amont de Caudebec, ces seuils portent les traces de l'érosion essentiellement par les dragages. Leur mise à nu et l'absence de sédiments mobiles sur des aires importantes de l'espace étudié est à mettre au compte (1) de l'indigence du matériel sédimentaire sableux disponible à l'amont du système, (2) de la dynamique exceptionnellement vive qui règne dans ce chenal (conditions macrotidales, débit fluvial excédant parfois $2000 \mathrm{~m}^{3} \mathrm{~s}^{-1}$ ). Elle est aussi imputable à la canalisation du chenal (dragages d'approfondissement, endiguements) et à son entretien par des dragages.

Les accumulations sableuses et graveleuses dues aux courants, identifiées en d'autres lieux du chenal, sont bien reconnues en domaine marin. Localement (berges internes de méandres), leur présence peut poser des problèmes pour la navigation. Enfin, d'autres échos sont particuliers à cet environnement estuarien canalisé et à l'intervention humaine, incluant dragages, aménagements des berges et du chenal.

\section{Remerciements}

Ces travaux ont été réalisés dans le cadre du Programme scientifique Seine-Aval, financé par les partenaires suivants : l'Etat, la Région Haute-Normandie et les autres Régions du Bassin Parisien, l'Agence de l'Eau Seine-Normandie et les industriels de Haute-Normandie.

\section{Références}

Auffret J.P., d'Ozouville L. (1986) Apports de l'imagerie foumie par le sonar à balayage latéral à la connaissance de la dynamique sédimentaire en Baie de Seine. Actes Coll. "La Baie de Seine" (GRECO-Manche), Caen, IFREMER-CNRS, 201-209.

Avoine J. (1981) L'estuaire de la Seine : sédiments et dynamique sédimentaire. Thèse de $3^{\text {ème }}$ cycle, Université de Caen, $236 \mathrm{pp}$.

Belderson R.H., Kenyon N.H., Stride A.H., Stubbs A.H. (1972) Sonographs of the Sea Floor. A picture atlas, Elsevier ed., Amsterdam, 185 pp.

Belderson R.H., Johnson M.A., Kenyon N.H. (1982) Bedforms. In : Offshore tidal sands, process and deposits, A.H. Stride ed., 27-57.

Berné S., Augustin J.M., Braud F., Chene G., Walker P. (1986) Cartographie et interprétation de la dynamique sédimentaire des plates-formes continentales : améliorations de la technique d'observation par sonar latéral. Bull. Soc. Géol. France, 8, t. II-3, 437-446.

D'Ollier B. (1979) in : Estuarine Hydrography Sedimentation, K. R. Dyer ed., Cambridge Univ. Press, 57-86.

Delouis A. (1997) Les dragages en Seine. Bulletin de liaison du Programme Scientifique SeineAval, 7, 4-5.

Duck R.W., McManus J. (1990) Uses of Side-Scan sonar in lakes and reservoirs. Proceedings $6^{\text {th }}$ Int. Ass. Engineering Geology, 6-10/08/1990, Amsterdam, 1080-1085.

Harris P.T., Collins M.B. (1984). Side-Scan Sonar investigation into temporel variations in sand waves morphology : Helwick sands, Bristol channel. Geo-Marine Letters, Vol. 4, 91-97. 